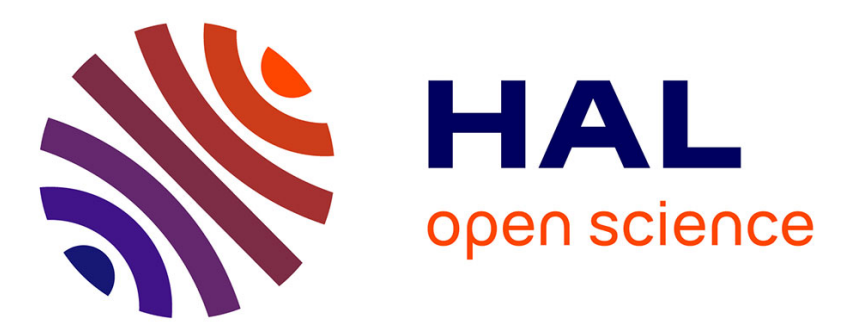

\title{
Nicolas de Francfort, un Allemand à Venise au tournant du XVe siècle
}

\author{
Catherine Kikuchi
}

\section{To cite this version:}

Catherine Kikuchi. Nicolas de Francfort, un Allemand à Venise au tournant du XVe siècle. Revue historique, 2014, 672. hal-01586399

\section{HAL Id: hal-01586399 \\ https://hal.science/hal-01586399}

Submitted on 12 Sep 2017

HAL is a multi-disciplinary open access archive for the deposit and dissemination of scientific research documents, whether they are published or not. The documents may come from teaching and research institutions in France or abroad, or from public or private research centers.
L'archive ouverte pluridisciplinaire HAL, est destinée au dépôt et à la diffusion de documents scientifiques de niveau recherche, publiés ou non, émanant des établissements d'enseignement et de recherche français ou étrangers, des laboratoires publics ou privés. 


\title{
Nicolas de Francfort, un Allemand à Venise au tournant du $\mathrm{XV}^{\mathrm{e}}$ siècle $^{1}$
}

\author{
CATHERINE KIKUCHI
}

L'historiographie de l'imprimerie vénitienne s'est longtemps construite à partir de biographies et d'annales typographiques d'imprimeurs ou d'ateliers. Plus récemment, Martin Lowry a rompu avec ce modèle : ses ouvrages dressent un tableau du monde économique et social dans lequel évoluaient les imprimeurs qu'il étudie, Nicolas Jenson et Alde Manuce, en dépassant le cas individuel pour comprendre le fonctionnement du milieu du livre vénitien ${ }^{2}$. Il s'agit d'élargir la réflexion à la société vénitienne et à sa plus ou moins grande perméabilité. Partant des réflexions qui ont été élaborées dans de nombreux travaux sur le rôle de la biographie dans les études historiques ${ }^{3}$, j'aimerais tenter d'apporter des éléments d'analyse sur la société vénitienne du tournant $d u X V^{e}$ siècle à partir du parcours d'un individu, un imprimeur d'origine allemande, Nicolas de Francfort (mort en 1524).

Pour reprendre les termes de Thierry Dutour, il s'agit, à partir de sources hétérogènes et dispersées, d'informer sur une existence sociale ${ }^{4}$ :

«Le problème est ici la mise en relation d'un individu avec ses contemporains pour retrouver des conduites spécifiques à une fonction, à une position dans une collectivité. Le concept central de la démarche est celui de rôle social ; le premier outil est la prosopographie $»^{5}$.

En effet, le rôle social d'un imprimeur vénitien, que nous nous proposons de retrouver, est à mettre en relation avec le début d'une étude prosopographique des imprimeurs et éditeurs vénitiens entre 1469 et le premier tiers du XVI siècle. Il s'agit d'aller au-delà de la simple carrière et d'aborder la totalité de la vie sociale et économique d'un imprimeur vénitien à partir du croisement de sources archivistiques variées.

Nicolas de Francfort est un personnage secondaire dans l'histoire de l'imprimerie européenne, alors que le récit que l'on fait de celle-ci s'est longtemps bâtie autour de l'étude de grandes figures mythifiées. C'est justement parce que Nicolas de Francfort est un personnage

$1 \quad$ Je tiens à remercier Mario Infelise pour ses conseils et ses indications archivistiques ; son article, Mario Infelise, «Franckfordia (da Francoforte), Nicolo », in Dizionario Biografico degli Italiani, Rome, Treccani, 1998, .a été le point de départ de cette étude. Je remercie également Élisabeth Crouzet-Pavan pour ses précieux avis et conseils. Je remercie enfin Florian Besson pour ses relectures et ses conseils. Certaines sources qui ont servi à cette étude ont pu être trouvées grâce à la Busta del Duca di Rivoli de l'Archivio di Stato di Venezia (ASV) qui recense les documents présents dans cette archive concernant les imprimeurs, libraires ou graveurs aux XV et XVI ${ }^{\mathrm{e}}$ siècles.

$2 \quad$ Martin Lowry, Nicholas Jenson and the Rise of Venetian Publishing and Renaissance Europe, Oxford, Blackwell, 1991; Martin Lowry, The World of Aldus Manutius: business and scholarship in Renaissance Venice, Ithaca, Cornell University Press, 1979.

3 On pense bien sûr à l'ouvrage de Jacques Le Goff, Saint Louis, Paris, Gallimard, 1996. Nous nous appuyons également sur les réflexions abordées dans le colloque Problèmes et méthodes de la biographie, Paris, 1985, Paris, Puf, 1985. Les apports de la sociologie sur ce sujet ont été importants, notamment avec l'article de Pierre Bourdieu, «L'Illusion biographique », Actes de la recherche en sciences sociales, 1986, vol. 62, n 62-63, pp. 69-73. On peut également se référer aux réflexions de Paul Ricœur dans Temps et Récit, Paris, Seuil, 1983, pp. 322-332.

4 Thierry Dutour, «L'Approche biographique des personnages secondaires : le cas de Guy VI de la Trémoille (1343-1397) », dans Problèmes et méthodes de la biographie, op. cit., pp. 23-32, p. 27.

5 Ibidem, p. 29. 
secondaire dans la grande histoire de l'imprimerie vénitienne qu'il permet de révéler certains des mécanismes à l'œuvre à Venise pour l'intégration d'un artisan étranger. Nous nous intéresserons donc à la construction et à l'élaboration de sa position sociale et professionnelle. Nicolas de Francfort imprime son premier ouvrage à Venise en 1473, en collaboration avec un autre imprimeur allemand, Franz Renner. On le connaît ensuite comme imprimeur ou éditeur jusqu'en 1516. Le dernier acte le mentionnant vivant est son testament du 23 décembre $1524^{6}$, et nous savons qu'il meurt très peu de temps après ${ }^{7}$. Cet individu est donc le témoin et l'acteur de la première époque de l'imprimerie vénitienne. La nouvelle technique est introduite en 1469 par l'imprimeur Johann de Spire, d'origine allemande ; celui-ci avait obtenu du Conseil un monopole de cinq ans. Johann de Spire mourant en 1470, la concurrence est relancée et une multitude d'ateliers typographiques ouvrent dans la ville. Entre 1469 et 1500, on compte 233 ateliers d'imprimerie ${ }^{8}$. Jusqu'au début des années 1480, l'imprimerie vénitienne est dominée par les Allemands, en particulier ceux impliqués dans les deux grandes compagnies de Johann de Cologne et de Nicolas Jenson ${ }^{9}$. Avec la mort de ces deux figures, l'imprimerie vénitienne du début des années 1480 semble passer plus directement aux mains d'imprimeurs d'origine italienne; le poids des Allemands devient relativement modeste ${ }^{10}$. Pourtant, alors qu'on ne trouve plus que très peu d'imprimeurs de grande envergure d'origine germanique entre 1480 et le début du XVI siècle, Nicolas de Francfort maintient son activité, même si celle-ci reste peu importante quantitativement.

Je développerai ici d'abord la question de l'installation et de la carrière professionnelle de Nicolas de Francfort à Venise en essayant de montrer quels sont les soutiens dont il a bénéficié et quelle a pu être sa position parmi les autres imprimeurs à Venise. J'aborderai également la question de sa production imprimée au cours de sa carrière. Par la suite, je m'intéresserai plus directement à son existence sociale et à ses alliances, en particulier matrimoniales; je m'efforcerai de montrer comment les choix d'appartenance à des confréries, les choix de mariages, ou d'investissements ont pu contribuer à l'inscrire dans la société vénitienne, en dehors du cercle restreint des imprimeurs ou libraires de la ville. Enfin, je terminerai par la situation de Nicolas de Francfort à sa mort en 1524 et je m'interrogerai sur l'ambivalence entre son origine allemande et son insertion vénitienne.

\section{LA CONSTRUCTION D’UNE POSITION PROFESSIONNELLE}

\section{L’arrivée à Venise : des liens étroits au sein de la colonie allemande}

On ne sait rien de Nicolas de Francfort avant son arrivée à Venise. Tout au plus pouvons-nous faire quelques suppositions. Nicolas de Francfort venait sans doute de Francfort-sur-le-Main,

$6 \quad$ ASV, Cancelleria inferiore, Miscellanea, b. 29, $\mathrm{n}^{\circ} 2936$.

$7 \quad$ Dans la mariegola de la scuola grande di S. Marco, on trouve après son nom la mention Mori 1524, ASV, Scuola grande di S. Marco, Atti, b. 4, Mariegola 1480-1549. Le calendrier vénitien faisant commencer la nouvelle année le $1^{\mathrm{er}}$ mars, la mort de Nicolas de Francfort a pu avoir lieu dans les derniers jours de décembre 1524, juste après l'établissement de son testament ou dans les deux mois qui ont suivi, au début de l'année 1525.

8 Paul Needham, «Venetian Printers and Publishers in the Fifteenth Century », in Luigi Baldamo (dir.), Anatomie bibliologiche, Florence, Olschki, 1999, section I.

$9 \quad$ Sur les débuts de l'imprimerie vénitienne, on peut se reporter au très clair article de Marino Zorzi, «Stampatori tedeschi a Venezia », in Venezia e la Germania : arte, politica, commercio, due civiltà a confronto, Milan, Electa, 1986, pp. 115-133.

$10 \quad$ Marino Zorzi, « Venezia e la Germania », art. cit., p. 132. 
comme de nombreux autres imprimeurs, éditeurs ou associés dans les premières années de l'imprimerie à Venise. S'il avait un nom de famille qu'il a abandonné à son arrivée, il ne faisait certainement pas partie du patriciat; il aurait pu appartenir à une famille marchande de Francfort, mais nous n'avons pas de traces de contacts directs avec les autres membres de l'élite francfortoise. Rien ne permet donc de déterminer précisément son statut dans sa ville d'origine $^{11}$. Il semble être un artisan allemand immigré à Venise comme il y en avait beaucoup dans toute l'Italie ${ }^{12}$.

Nicolas de Francfort est arrivé à Venise où il a pu se lier facilement avec d'autres immigrés ou résidents d'origine germanique ${ }^{13}$. La colonie allemande a été un lieu d'ancrage social et économique extrêmement important pour les premiers imprimeurs vénitiens. Au centre de cette communauté et de ses activités économiques se trouve le Fondaco dei tedeschi, à la fois « hôtel, entrepôt, bourse aux affaires où les Allemands étaient installés depuis deux siècles et demi $\gg$. Au-delà, on trouve de nombreux Allemands parmi les boulangers et les cordonniers, mais aussi dans les métiers du métal et les marchands de papier. Philippe Braunstein a pu constater des liens étroits entre les nouveaux immigrants à Venise et les anciens, parfois devenus citoyens vénitiens ${ }^{15}$. Comme Johann de Spire, Nicolas de Francfort a pu profiter de ces sociabilités et y trouver des capitaux, du savoir-faire technique et des partenaires. On le constate d'abord en voyant apparaître Nicolas de Francfort dans de nombreux actes impliquant d'autres Allemands et en particulier d'autres Allemands du monde du livre ${ }^{16}$. Son

11 En particulier, il ne peut y avoir d'identification certaine avec les noms présents dans les livres de bourgeoisie de Francfort.

12 On pense en particulier aux travaux sur les Allemands à Florence ou Rome, mais également à une échelle plus large, à l'étude des migrations artisanales allemandes en Europe : Clifford William Maas, The German Community in Renaissance Rome, 1378-1523, Rome, Herder, 1981 ; Knut Schulz, «Deutsche Handwerkergruppen in Italien, besonders in Rom (14.-16. Jh)» dans Le Migrazioni in Europa secc. XIII-XVIII, Prato, 1993, Florence, Le Monnier, 1994, pp. 567-591 ; Lorenz Böninger, Die Deutsche Einwanderung nach Florenz im Spätmittelalter, Leyde, Brill, 2006.

13 La question de ce qu'est un Allemand ou l'Allemagne au $\mathrm{XV}^{\mathrm{e}}$ siècle a été abordée par de nombreux ouvrages récents, notamment Cecilie Hollberg, Deutsche in Venedig im Späten Mittelalter : eine Untersuchung von Testamenten aus dem 15. Jahrhundert, Göttingen, V\&R Unipress, 2005. Mais la question a été particulièrement traitée par Philippe Braunstein dans ces deux articles : Philippe Braunstein, «Appunti per la storia di una minoranza: la popolazione tedesca di Venezia nel Medioevo », in Rinaldo Comba, Gabriella Piccini, Giuliano Pinto (dir.), Strutture familiari, epidemie, migrazioni nell'Italia medievale, Naples, Edizioni scientifiche italiane, 1984, pp. 511-517; Philippe Braunstein, «Remarques sur la population allemande de Venise à la fin du Moyen-Âge », dans Venezia, centro di mediazione tra Oriente e Occidente (secoli XV-XVI). Aspetti e problemi, Venise, 1973, Florence, 1977, pp. 133-143. Il en ressort que cela a bien un sens de parler d'Allemands à Venise, parce que cette communauté a été définie par Venise notamment à travers le Capitulaire du Fondaco, et parce que cette communauté est soudée par des solidarités et des intérêts communs ainsi que par une unité culturelle et linguistique, allant des pays de l'Est de l'Empire, à l'Allemagne actuelle et à la Flandre.

14 Philippe Braunstein, «Les Allemands et la naissance de l'imprimerie vénitienne », Revue d'études italiennes, 1981, vol. 27, n 4, p. 384. Sur le Fondaco, Henry Simonsfeld, Der Fondaco dei Tedeschi in Venedig und die Deutsch-Venetianischen Handelsbeziehungen, Stuttgart, J. G. Cotta, 1887 ; Karl-Ernst Lupprian, Il Fondaco dei tedeschi e la sua fonzione di controlle del commercio tedesco a Venezio, Venise, Centro Tedesco di Studi Veneziani, 1978 ; Gerhard Rösch, «Il Fondaco dei Tedeschi », in Venezia e la Germania, op. cit., pp. 5172 ; Carolin Wirtz, " "Mercator in fontico nostro". Mercanti tedeschi fra a Germania e il Fondaco dei Tedeschi a Venezia », dans Presenze tedesche a Venezia, Venise, 2002/2003, Rome, Edizioni di storia e letteratura, 2005, pp. 1-48.

15 Philippe Braunstein, «Les Allemands et la naissance de l'imprimerie vénitienne », art. cit., pp. 384385.

16 Il est l'exécuteur testamentaire de Johann Herbort, associé de Johann de Cologne (ASV, Cancelleria inferiore, Notai, b. 124, f $117 \mathrm{v}, 4$ octobre 1484), de l'imprimeur Benedetto Fontana, fils de Franz Renner (ASV, 
rôle récurrent d'exécuteur testamentaire témoigne de liens forts : c'est une tâche qui était souvent confiée aux membres de la famille proche. Il est courant de nommer également des membres du même métier et des amis, mais le testateur doit pouvoir leur faire suffisamment confiance pour leur remettre cette responsabilité.

Plus particulièrement, Nicolas de Francfort semble avoir eu des relations étroites avec la famille Liechtenstein; il est exécuteur testamentaire de Hermann, imprimeur, et Peter Liechtenstein, neveu de Hermann lui-même imprimeur et éditeur, est exécuteur testamentaire de Nicolas de Francfort en $1519^{17}$. Il est surtout mentionné comme son compère, ce qui signale un lien électif fort qui peut être fréquent entre membres d'un même métier. Les relations de parenté spirituelle permettaient ainsi à des collaborateurs ou à des confrères de renforcer des rapports purement professionnels par des liens à la fois religieux et familiaux. C'est le cas à Florence, où il est fréquent de demander à des collègues, voire à toute la corporation de parrainer un seul enfant ${ }^{18}$. De plus, la parenté spirituelle utilisée pour sanctionner et garantir rituellement des relations d'affaires a notamment été bien observée pour la Vénétie aux XVI ${ }^{\mathrm{e}}$ et XVII ${ }^{\mathrm{e}}$ siècles ${ }^{19}$.

D'autres documents laissent penser que Nicolas de Francfort pouvait rendre des services aux autres imprimeurs vénitiens, bien qu'il n'ait pas directement conclu de partenariat avec eux ${ }^{20}$. Il semble bien être parvenu à tisser des liens d'amitié, de confiance et de réciprocité qui ont pu l'aider dans ses débuts à Venise et également dans la suite de sa carrière. Un recours et une personne de confiance pour ses collègues ou ses compatriotes à Venise : cette image semble correspondre également à son parcours en tant qu'imprimeur et éditeur.

\section{Les débuts de l'activité de Nicolas de Francfort : des alliances dans la colonie allemande}

Nicolas de Francfort a débuté son activité en s'associant avec Franz Renner de Hailbrunn ${ }^{21}$. Les colophons de leurs ouvrages sont relativement peu explicites, si ce n'est la mention «Franciscus de Hailbrunn et Nicolas de Francfort associés ${ }^{22}$, qui signale l'association durable qui a existé entre eux. Nous n'avons pas de traces du contrat de société qui a pu être passé entre ces imprimeurs et les colophons ne permettent pas de déterminer la répartition éventuelle des tâches entre eux. Ils continuent à imprimer en tant qu'associés jusqu'en 1477.

Notarile, Testamenti, b. 51, $\mathrm{n}^{\circ}$ 91, 6 avril 1492), de l'imprimeur Hermann Liechtenstein (ASV, Notarile, Testamenti, b. 66, $\mathrm{n}^{\circ} 8$ et $\mathrm{n}^{\circ} 8$ bis, 28 juin 1494) et du marchand Conrad de Salsbourg (ASV, Notarile, Testamenti, b. 295, $\mathrm{n}^{\circ} 29,21$ juin 1501).

$17 \quad$ ASV, Notarile, Testamenti, b. 66, n 320.

18 Christiane Klapisch-Zuber, «Compérage et clientélisme à Florence (1360-1520) », Ricerche storiche, vol. 15, $\mathrm{n}^{\circ}$ 1, 1985, pp. 61-76, cité dans Guido Alfani et Vincent Gourdon, « Il ruolo economico del padrinato : un fenomo osservabile », in Guido Alfani (dir.), Il ruolo economico della famiglia, Rome, Bulzoni, 2006, pp. 129-177, p. 162.

19 Guido Alfani et Vincent Gourdon, «Il ruolo economico del padrinato : un fenomo osservabile », art. cit., p. 158 .

20 Antonio Sartori, Documenti padovani sull'arte della stampa nel sec. XV, Padoue, Tipografia Antoniana, 1960, pp. 217-220, doc. 87.

$21 \quad$ Au sujet de l'identification de cet imprimeur, voir Robert Ridolfi, «Francesco della Fontana, stampatore e libraio a Venezia », dans Studi bibliografici. Atti del convegno dedicato alla storia del libro italiano nel V centenario dell'introduzione dell'arte tipografica in Italia, Bolzano, 1965, Florence, Olschki, 1967.

22 Franciscus de Hailbrun et Nicolaus de Franckfordia socii, par exemple dans Leonardus de Utino, Sermones quadragesimales de legibusdicti, Venise: Franciscus Renner, de Heilbronn avec Nicolaus de Francfort, 1473. Folio. $\mathrm{N}^{\circ}$ ISTC : i100143000, cité dans Georg Wolfgang Panzer, Annales typographici, Hildesheim, G. Olms, 1963, vol. 3, n 122. 
Par la suite, les collaborations de Nicolas de Francfort dans ses premières années d'activité se dirigent exclusivement vers la colonie allemande, en collaborant avec Leonard Wild et les associés Erhard Ratdolt, Peter Löslein et Bernhard Maler. Dans les deux cas, il s'agit d'éditions réalisées aux frais de Nicolas de Francfort, à savoir sumptibus et jussu ou bien expensis Nicolas de Franckfordia selon les colophons ${ }^{23}$. Dès 1478, celui-ci travaille donc comme éditeur $^{24}$, en apportant les fonds nécessaires à la publication d'un ouvrage et en collaboration exclusive avec des typographes allemands ${ }^{25}$. En se basant sur ces premières années, on a pu penser que Nicolas de Francfort était uniquement éditeur, sans connaissance technique de l'imprimerie. En effet dans les années 1470, Nicolas de Francfort semble plutôt concerné par le financement et l'organisation commerciale que par la pratique de l'imprimerie. Il a pu profiter de son association avec Renner, qui semble s'être montrée relativement productive et fructueuse - seize éditions de textes religieux de large diffusion en quatre ans. Cette activité a pu être suffisamment rentable pour qu'il ait pu par la suite financer d'autres éditions avec d'autres imprimeurs, malgré le fait qu'il n'était actif à Venise que depuis cinq ans.

Nicolas de Francfort a donc pu compter sur des alliances et des appuis au sein de la colonie allemande pour trouver sa place dans le milieu extrêmement instable de l'imprimerie vénitienne. Les relations qu'il a nouées avec ces imprimeurs, facilitées sans doute par leur appartenance commune à la colonie allemande à Venise, lui ont permis de stabiliser sa position dans la ville par des associations plus ou moins durables. Malgré tout, les années suivant la fin de l'association avec Renner sont des années bien moins productives qu'auparavant : on ne connaît que trois éditions le mentionnant entre 1478 et 1480. Il retrouve un rythme de publication plus soutenu à partir de 1481, date à laquelle son nom apparaît seul dans les colophons.

\section{La construction progressive de sa renommée et de sa capacité d'investissement}

Sans doute grâce au capital qu'il avait pu accumuler depuis le début de son activité, et peutêtre grâce à un apport d'autres revenus, Nicolas de Francfort imprime seul pendant neuf ans. Entre 1481 et 1489, apparaissent dans les colophons de ses éditions des mentions laissant penser qu'il maîtrise lui-même l'art d'imprimer : il s'agit essentiellement de références à son ars, selon différentes variantes, mais qui signalent généralement bien un typographe, et pas

\footnotetext{
23 Ainsi, dans Biblia latina, Venise : Leonardus Wild pour Nicolaus de Francfort, [juillet] 1478. Folio. $\mathrm{N}^{\circ}$ ISTC : ib00558000, cité dans Ibidem, $\mathrm{n}^{\circ} 350$; ou dans Breviarium Romanum, Venise : Bernhard Maler, Erhard Ratdolt, Peter Löslein pour Nicolaus de Francfort, 1478. Octavo. N $^{\circ}$ ISTC : ib01118100, Staatsbibliothek München, Ink B-888GW 5147. Les colophons sont de courts textes, insérés à la fin de l'ouvrage, qui faisaient office de publicité, à la fois pour l'édition et pour l'atelier typographique en tant que tel. Ils mentionnent le plus souvent l'imprimeur, l'éditeur parfois, le lieu et la date, et contiennent occasionnellement un éloge de l'auteur ou de l'imprimeur. L'avance des fonds est généralement signalée par les termes expensis ou impensis ; l'impression à proprement parler, par les termes ars, opera ou éventuellement cura.

$24 \quad$ Il n'existe qu'un seul mot en français, éditeur, pour désigner à la fois celui qui avance les fonds et celui qui effectue le travail d'édition du texte, là où l'anglais a d'une part publisher et d'autre part editor. Ici, le terme d'éditeur fera référence à la fonction d'avance d'argent.

25 On a confirmation de ce rôle d'éditeur à travers le contrat que Nicolas de Francfort passe avec Leonard Wild le 14 mars 1478 : texte publié dans Rinaldo Fulin, «Documenti per servire alla storia della tipografia veneziana », Archivio Veneto, vol. XXIX, 1882, pp. 84-212, doc. 2.
} 
seulement un éditeur ${ }^{26}$. On peut alors supposer soit qu'il a effectivement appris lui-même la technique de l'imprimerie, soit qu'il est parvenu à monter un atelier typographique autonome. Celui-ci est peut-être sous la direction d'un autre typographe mais qui imprime sous le nom de Nicolas de Francfort. Cette deuxième solution semble la plus probable, surtout si l'on considère la suite de son activité, puisqu'il reprend un strict rôle d'éditeur à partir des dernières années du $X v^{\mathrm{e}}$ siècle.

À partir de ses colophons, de la fin des années 1480 jusqu'à la fin de son activité éditoriale, Nicolas de Francfort tente également de consolider sa réputation et de faire en sorte que son nom ne se perde pas au milieu du flot d'éditions qui envahissent le marché italien et européen. Il faut pour ces imprimeurs que le nom même de l'atelier devienne une garantie de qualité et de correction. Les colophons servent alors à construire sa réputation, en s'affirmant très compétent afin que les lecteurs associent progressivement ce nom à des livres de bonne qualité. Cependant, les dates auxquelles ces colophons apparaissent dans la carrière de Nicolas de Francfort laissent penser que celui-ci possède déjà une réputation suffisamment solide pour pouvoir s'affirmer sans ridicule famosissimus ou probatissimus ${ }^{27}$. Ce changement de politique publicitaire correspond à une période où Nicolas de Francfort travaille en collaboration avec d'autres imprimeurs mais de façon bien plus espacée que les années précédentes. Entre 1490 et 1500 , on ne compte que neuf éditions le mentionnant ${ }^{28}$. De plus, selon les colophons, Nicolas de Francfort se contente pour ces éditions de l'avance des frais. Après 1500, son activité est encore plus restreinte. On sait qu'il imprime un ouvrage en 1511 ; on lui connaît également une édition en $1516^{29}$.

Entre 1490 et sa mort, Nicolas de Francfort semble donc se détourner de l'imprimerie, n'éditant des livres qu'occasionnellement, avec seulement trois partenaires privilégiés, dont deux Allemands, Johann Emericus de Spire et Johann Hamman ${ }^{30}$, alors même que les Allemands se font plus rares parmi les imprimeurs vénitiens et pèsent moins sur le marché du livre. Les éditions qu'il réalise avec ces partenaires sont en réalité des investissements ponctuels que Nicolas de Francfort mène en parallèle avec d'autres activités. L'imprimerie n'est plus son activité principale, ce d'autant plus que vers 1500 il commence certainement à être assez âgé. Néanmoins, sa présence continue sur le marché en tant qu'éditeur témoigne de l'aisance ainsi que de la réputation qu'il a pu acquérir pendant ses années de plein exercice. Le changement de présentation dans ses colophons peut également signaler une position assurée dans le monde de l'imprimerie vénitienne, alors même qu'il n'a plus besoin de produire des livres de façon continue. Il n'a pas été évincé du monde de l'imprimerie par une

\footnotetext{
26 Par exemple dans Missale Romanum, Venise : Nicolaus de Francfort, 1484. Octavo. $\mathrm{N}^{\circ}$ ISTC : im0069300 : «Explicit missale secundum morem romane ecclesie. Impressum Venetiis arte et impensis Nicholai de Franckffordia Anno domini MCCCCLXXXIIII Deo gratias ». Cité dans Georg Wolfgang Panzer, Annales typographici, op. cit. $\mathrm{n}^{\circ} 761$.

27 L'impression est dite avoir été réalisée sumptibus et iussu famosissimi Nicholai de Francofordia dans Missale Romanum, Venise : Johannes Hamman pour Nicolaus de Francfort, $1^{\text {er }}$ juillet 1493 . Octavo. $\mathrm{N}^{\circ}$ ISTC : im00708000, cité dans Ibidem, $\mathrm{n}^{\circ}$ 1679. Il est également décrit comme probatissimus vir dans Antonius de Bitonto, Sermones dominicales per totum annum, Venise: Bonetus Locatellus pour Nicolaus de Francfort, 10 octobre 1499. Octavo. $\mathrm{N}^{\circ}$ ISTC : ia00894000, cité dans Ibidem, n 2444.

28 Selon l'Incunabula Short Title Catalogue et le Gesamtkatalog der Wiegendrucke, consultés d'après leur site internet.

$29 \quad$ Ester Pastorello, Tipografi, editori, librai a Venezia nel secolo XVI, Florence, Olschki, 1924, $\mathrm{n}^{\circ} 196$.

30 Paul Needham, «Venetian Printers and Publishers in the Fifteenth Century », art. cit., section I, $n^{\circ} 99$, $n^{\circ} 138, n^{\circ} 139$ et section III.
} 
faillite ou une mauvaise gestion qui lui aurait fait perdre à fois argent et crédit auprès de ses pairs. Son retrait progressif, mais pas total avant la fin des années 1510, montre au contraire qu'il reste un soutien financier possible et un partenaire fiable.

Sa position relative parmi les imprimeurs ne doit donc pas seulement s'évaluer en fonction de sa production quantitative, assez faible. L'analyse chronologique a pu révéler différentes phases dans sa carrière, qui témoignent d'une assise économique certaine et construite par son activité éditoriale ; cette stabilité lui permet de rester un acteur en vue dans le monde du livre vénitien.

\section{Une production atypique à Venise}

Afin de compléter cette vue d'ensemble de la carrière de Nicolas de Francfort, il convient de s'intéresser plus précisément à sa production. Comme on l'a déjà dit, celle-ci est faible comparée aux grandes entreprises typographiques qui lui sont contemporaines. En regroupant les éditions qu'il a financées et celles dans lesquelles il a eu une part active, on compte 51 éditions de 1473 jusqu'en $1500^{31}$. On est bien loin des 45 éditions en trois ans de Nicolas Jenson $^{32}$.

Par ailleurs, le contenu même de sa production est très atypique pour une ville comme Venise. Celle-ci est constituée quasi-exclusivement de textes religieux, à trois exceptions près : trois ouvrages de philosophie ou d'histoire qu'il a imprimés avec Franz Renner, sans doute dans les premières années de leur collaboration, même si les dates pour deux d'entre elles ne sont pas certaines $^{33}$. Pour le reste, la production de Nicolas de Francfort, que ce soient les ouvrages qu'il a publiés seul ou ceux qu'il a édités avec d'autres, sont exclusivement des ouvrages religieux : bréviaires et missels, recueils de sermons, sommes théologiques, Bibles, hagiographies...

Tous les imprimeurs de cette époque avaient une production de textes religieux : ceux-ci étaient généralement rapidement vendus à un large public et pouvaient être très rentables. Cependant, si l'on se réfère aux études des productions imprimées qui ont pu être effectuées à l'échelle de l'Europe et de Venise, on constate qu'une telle spécialisation détonne dans le paysage éditorial italien. En effet, la production de textes religieux en Europe s'élève à $38 \%$ de la production imprimée totale, mais avec une forte différenciation entre l'Italie et le reste de l'Europe: en Italie, les imprimeurs ne produisent que $28 \%$ de textes religieux, alors qu'hors-Italie, cette part atteint $48 \%$. La production religieuse de Venise est encore un peu plus réduite puisqu'elle s'élève seulement à $26 \%$. Certes, elle y reste la deuxième production la plus importante derrière la littérature, vaste domaine regroupant à la fois les classiques et la littérature vernaculaire, mais elle n'occupe pas une place aussi importante qu'ailleurs en Europe.

31 Selon le Gesamtkatalog der Wiegendruck ; 49 selon l'ISTC. On a un relevé presque systématique pour la période incunable. Celui-ci est plus lacunaire pour le $\mathrm{XVI}^{\mathrm{e}}$ siècle.

32 Marino Zorzi, « Venezia e la Germania », art. cit., p. 129.

33 Il s'agit de Antonius Andreae, Quaestiones super XII libros Metaphysicae Aristotelis, Venise: Franciscus Renner, de Heilbronn et Nicolaus de Francfort, vers 1473-1477. Folio. N ISTC : ia0057900; de Lucius Fenestella, De Romanorum magistratibus, Venise: Franciscus Renner, de Heilbronn, et Nicolaus de Francfort, vers 1473-1477. Quarto. № ISTC : if00060000; et de Johannes de Janduno, Quaestiones super libros De anima Aristotelis, Venise : Franciscus Renner, de Heilbronn, et Nicolaus de Francfort, 1473. Folio. $\mathrm{N}^{\circ}$ ISTC: ij00351000.

$34 \quad$ John M. Lenhart, Pre-Reformation Printed Books: A Study in Statistical and Applied Bibliography, New York, J. F. Wagner, 1935, p. 83. cité dans Leonardas V. Gerulaitis, Printing and Publishing in Fifteenth Century Venice, Londres, American Library Association, 1976, p. 62. 
Or il semble que les textes liturgiques en particulier aient été considérés comme un investissement rentable à terme. Achetés par des institutions, ils sont plus luxueux et l'Église est capable d'en payer un bon prix ${ }^{35}$. On en compte 64 éditions à Venise, dont 21 réalisées par Nicolas de Francfort, en particulier 17 dont l'édition a été réalisée par lui seul entre 1477 et 1489. Il a donc réalisé ou participé à la production de près d'un tiers des éditions liturgiques vénitiennes. Nicolas de Francfort semble être devenu le spécialiste vénitien des livres liturgiques $^{36}$. En collaboration avec d'autres, il a élargi ses publications à d'autres types d'ouvrages religieux, particulièrement la théologie ${ }^{37}$ et les sermons ${ }^{38}$.

Cette spécialisation est très atypique dans le paysage typographique vénitien et italien. Mais elle se justifie en termes économiques et explique aussi le nombre plus restreint d'éditions réalisées : pour ces ouvrages, les tirages sont généralement plus importants et plus coûteux, les volumes sont plus épais et nécessitent plus de papier et de main-d'œuvre. Enfin, cela pourrait expliquer la quasi-absence de partenaires italiens pour Nicolas de Francfort, si l'on excepte Bonetus Locatellus, un prêtre, les autres imprimeurs préférant une plus grande variété d'ouvrages.

La rentabilité des ouvrages liturgiques supposait malgré tout que Nicolas de Francfort dispose de débouchés importants. Cristina Dondi fait l'hypothèse que les imprimeurs vénitiens, tels que Nicolas Jenson ou Johann de Cologne, imprimaient sur commande des confraternités. Être membre de celles-ci leur permettait d'avoir accès à un marché assuré et de stimuler la demande du public ${ }^{39}$. Or Nicolas de Francfort ainsi que sa femme appartiennent à de très nombreuses confraternités ou scuole vénitiennes. Si l'on considère les deux testaments de Nicolas de Francfort, l'un du 25 mai $1519^{40}$, l'autre du 23 décembre $1524^{41}$, on constate qu'il fait des dons à plus d'une dizaine de scuole différentes ${ }^{42}$. Quant à sa femme, Andriana, d'après son testament du 22 mai $1519^{43}$, elle fait également des dons à cinq scuole différentes, qui sont comprises dans celles citées par son mari. Si l'on suit la démonstration de Cristina Dondi, l'adhésion à ces confréries aurait eu un rôle commercial et économique en vue de vendre les ouvrages imprimés ou financés par l'imprimeur, stratégie qui aurait été suivie par de nombreux autres imprimeurs vénitiens au $\mathrm{XV}^{\mathrm{e}}$ siècle.

$35 \quad$ Ibidem, p. 101.

36 Ajoutons que l'intérêt esthétique ou artistique de ces ouvrages ne semble pas avoir marqué les spécialistes ou les bibliophiles : Duc de Rivoli, Les Missels imprimés à Venise de 1481 à 1600, Paris, Rothshild, 1896 , p. 60.

37 Il imprime sept éditions d'ouvrages théologiques avec Franz Renner, une avec Leonard Wild, une avec Bonetus Locatellus et enfin une seul sans autre partenaire.

$38 \quad$ Il imprime trois éditions de sermons avec Franz Renner, une seul, deux avec Bonetus Locatellus et trois avec Johann Hamman.

39 Cristina Dondi, «Printers and Guilds in Fifteenth-Century Venice », La Bibliofilia, vol. 106, 2004, pp. 245-251.

$40 \quad$ ASV, Notarile, Testamenti, b. 66, $\mathrm{n}^{\circ} 320$.

$41 \quad$ ASV, Cancelleria inferiore, Miscellanea, b. 29, n 2936.

42 Ce sont les mêmes dans les deux testaments, à l'exception de la scuola de Santa Maria à S. Salvador, qui n'est mentionnée que dans le deuxième. Ces scuole sont représentées sur la carte en annexe, à l'exception d'une seule qui n'a pas pu être identifiée avec certitude. Celle-ci présente également les principales paroisses dont il sera question ici. Je remercie vivement ici Pascal Vuillemin qui m'a très aimablement fourni cette carte. On ne pouvait faire partie que d'une seule scuola grande, mais le fait de faire partie d'une scuola grande et de plusieurs scuole piccole ne semble pas poser problème : Brian Pullan, « Natura e carattere delle Scuole », in Terisio Pignatti (dir.), Le Scuole di Venezia, Milan, Electa, 1981, pp. 9-26.

$43 \quad$ ASV, Notarile, Testamenti, b. 66, $\mathrm{n}^{\circ} 12$. 
Cette hypothèse est tout à fait probable mais il faut la combiner avec d'autres réalités : il serait faux de voir dans l'adhésion à ces scuole une simple stratégie économique. À la différence de Nicolas Jenson, qui ne mentionne pas les confréries dans son testament et semble s'en être désintéressé à la fin de sa vie, pour Nicolas de Francfort, il ne faut pas exclure que ces adhésions multiples puissent témoigner d'une foi et d'une pratique religieuse approfondies, intenses. Celles-ci pourraient également avoir influencé sa production éditoriale religieuse. Au-delà des intérêts purement économiques, il faut également comprendre quel rôle ont pu jouer ces adhésions aux scuole dans la carrière et l'insertion sociale de Nicolas de Francfort à Venise.

\section{DES ALLIANCES EN DIRECTION DE LA SOCIETE VENITIENNE}

\section{Les scuole : tentative de regroupement et ouverture vers Venise}

La participation de Nicolas de Francfort aux confréries vénitiennes était tout à fait exceptionelle, si l'on en croit les legs de ses testaments. Si Leonardo Loredan, doge de Venise entre 1501 et 1521 , était membre d'à peu près cent vingt scuole ${ }^{44}$, l'appartenance à plus d'une dizaine de ces confréries reste rare dans le milieu des artisans, aussi aisés soient-ils.

La fonction d'intégration de ces scuole, leur fonction de socialisation et d'assistance ont été soulignées à de nombreuses reprises, notamment dans le travail fondateur de Brian Pullan sur les scuole grandi ${ }^{45}$. Dans le cas de Nicolas de Francfort, celui-ci appartient à la prestigieuse scuola grande de S. Marco, avec Nicolas Jenson ${ }^{46}$. D'après le copiste chargé de l'enregistrement des nouveaux confrères, il semble y avoir été admis vers 1484. Les scuole grandi à la fin du Moyen Âge, comme le montre l'étude de Brian Pullan, sont l'expression publique de la piété vénitienne ${ }^{47}$. Ce sont entre autres des institutions caritatives: les confrères forment deux groupes, les riches bienfaiteurs de la scuola et les pauvres qui en sont les bénéficiaires ${ }^{48}$. Dans le cas de Nicolas de Francfort et de Nicolas Jenson, ils sont tous deux asenti ala disciplina, c'est-à-dire la catégorie la plus aisée de la confrérie, ce qui leur procure un prestige social indéniable. Cela confirme également qu'en 1484, Nicolas de Francfort avait acquis des biens et une réputation suffisants pour pouvoir entrer dans cette confrérie en tant que membre bienfaiteur.

La scuola grande de S. Marco a également une utilité pratique pour Nicolas de Francfort. Il s'agit d'un véritable club social où il pouvait rencontrer les membres de l'élite vénitienne, des patriciens mais également d'autres membres de l'artisanat qualifié et enrichi de la ville. Martin Lowry avait souligné ce rôle dans le cadre de la scuola de S. Girolamo qui regroupait parmi ses membres un très large éventail de compétences ${ }^{49}$. Ici, une scuola grande est une porte d'entrée vers l'élite de la société vénitienne. De fait, la confrérie de S. Marco semble

$44 \quad$ Brian Pullan, « Natura e carattere delle Scuole », art. cit., p. 14.

45 Brian Pullan, Rich and Poor in Renaissance Venice: the Social Institutions of a Catholic State, to 1620, Oxford, Blackwell, 1971. Les sources relatives aux scuole piccole ont été inventoriées par Gastone Vio, Le Scuole piccole nella Venezia dei Dogi. Note d'archivio per la storia delle confraternite veneziane, Vicence, Colla, 2004. Enfin, on peut consulter l'ouvrage de Francesca Ortalli, Per Salute delle anime e delli corpi : scuole piccole a Venezia nel tardo Medioevo, Venise, Marsilio, 2001.

46 ASV, Scuola grande di S Marco, Atti, b. 4, Mariegola 1480-1549, f ${ }^{\circ}$ 100r. Pour Jenson, cité par Martin Lowry, Nicholas Jenson, op. cit., p. 112.

$47 \quad$ Brian Pullan, Rich and Poor in Renaissance Venice, op. cit., p. 51.

$48 \quad$ Ibidem, pp. 63-66.

49 Martin Lowry, « The Social World of Nicholas Jenson and John of Cologne », art. cit., p. 209. 
avoir eu une signification importante pour l'imprimeur et sa famille : son beau-fils, Jacomo, en fait partie en tant que confrère ordinaire; par ailleurs, Nicolas de Francfort et toute sa famille sont enterrés dans le caveau de la scuola ${ }^{50}$.

En ce qui concerne les autres scuole mentionnées dans les testaments de Nicolas de Francfort, on constate qu'il s'en trouve un peu partout dans la ville ${ }^{51}$. Si l'on tient compte de ses changements de résidence, on constate que son adhésion à certaines d'entre elles a été largement déterminée par le voisinage. Le cadre paroissial a son importance pour les pratiques dévotionnelles comme sociales, tout particulièrement pour les nouveaux arrivants qui s'y attachent davantage que ne peuvent le faire les Vénitiens installés, à une époque où la paroisse perd de sa cohésion ${ }^{52}$. Nicolas de Francfort appartient ainsi à deux scuole situées dans la paroisse de S. Marziale où il habite depuis 1494 au moins ${ }^{53}$. Un autre groupe de scuole semble avoir été déterminé par leur proximité avec son premier lieu de résidence, la paroisse de S. Canciano ${ }^{54}$ et leur proximité avec le quartier des imprimeurs et des Allemands. Ceux-ci semblent avoir investi les confréries des églises de S. Bartolomeo et de S. Salvador où ils occupent des postes de gouvernement de la scuola ${ }^{55}$. En particulier, la scuola du Rosaire de S. Bartolomeo, fondée en 1506, est la confrérie nationale des Allemands à Venise ${ }^{56}$.

Sans compter l'aspect religieux et de piété personnel que nous mentionnions plus haut, Nicolas de Francfort semble donc avoir mis à profit l'appartenance à des scuole de plusieurs manières, à la fois pour s'intégrer à la société vénitienne à travers une instance de socialisation reconnue, et à la fois pour conserver des liens de solidarité avec la communauté allemande. Les scuole nationales ou très investies par une nation donnée permettent de resserrer des liens professionnels ou de solidarité entre des immigrés à Venise, soumis parfois à des conditions sociales et économiques difficiles. Par ailleurs, les scuole piccole sans affiliation nationale particulière permettent de tisser des liens au niveau local et de s'insérer dans le réseau paroissial à l'échelle du quartier et de ses environs. Enfin, les scuole grandi offrent à leurs membres privilégiés un accès à la sociabilité de l'élite vénitienne, permettant

50 ASV, Cancelleria inferiore, Miscellanea, b. 29, $\mathrm{n}^{\circ} 2936,23$ décembre 1524; ASV, Cancelleria inferiore, Miscellanea, b. 29, $\mathrm{n}^{\circ} 2950,13$ avril 1528. L'inscription du tombeau est sans doute celle retrouvée par Henry Simonsfeld, Der Fondaco dei Tedeschi in Venedig und die Deutsch-Venetianischen Handelsbeziehungen, op. cit., $\mathrm{n}^{\circ} 63$ : il s'agit de la première épouse de Nicolas de Francfort, mais le nom de Fontana est également présent sur les relevés épigraphiques.

$51 \quad$ Outre les indications de son testament, le nom de Nicolas de Francfort est présent en particulier dans les mariegole des scuole de S. Christophoro et de Santa Caterina : ASV, Scuole piccole e suffragi, b. $406, \mathrm{f}^{\circ} 59$ et Museo Correr, Mariegola $\mathrm{n}^{\circ}$ 119, Scuola di Santa Caterina, $\mathrm{f}^{\circ}$ 52. Ces listes ont le plus souvent été perdues pour les autres confréries.

52 Comme l'a montré Élisabeth Crouzet-Pavan, même si cette forme de sociabilité connaît un affaiblissement à la fin du XV ${ }^{\mathrm{e}}$ siècle : Élisabeth Crouzet-Pavan, «Sopra le Acque salse » : espaces, pouvoir et société à Venise à la fin du Moyen Âge, Rome, Istituto storico italiano per il Medio Evo, 1992, vol. 1, p. 597.

53 On trouve une unique source le faisant habiter à SS. Apostoli (Museo Correr, Mariegola $\mathrm{n}^{\circ} 118, \mathrm{f}^{\circ}$ 52), mais cette paroisse jouxtant celle de S. Canciano, on peut considérer qu'il s'agit d'une imprécision ou d'une erreur.

$54 \quad$ ASV, Scuola grande di S Marco, Atti, b. 4, Mariegola 1480-1549, f ${ }^{\circ}$ 100r.

55 Gerhard Rösch, «Il Fondaco dei Tedeschi », art. cit; , p. 62. et Francesca Ortalli, Per Salute delle anime e delli corpi, op. cit., p. 106 et ASV, Provveditori di Comun, reg. T, f ${ }^{\circ} 290-291$.

$56 \quad$ Le terme de «national» n'a pas le sens qu'il a pu prendre au XIX ${ }^{\mathrm{e}}$ siècle mais a ici le sens des nationes, à l'image de celle que l'on retrouve dans les universités médiévales. Voir sur ce sujet le congrès de 2013 de la SHMESP portant sur « Nation et nations au Moyen Âge », à paraître. 
de côtoyer des membres des couches les plus aisées, favorisées et les mieux insérées économiquement et socialement.

Pour terminer cette analyse, nous pouvons reprendre les réflexions d'Élisabeth Crouzet-Pavan appliquées à la paroisse de S. Barnaba, qui permettent de mieux comprendre cet ancrage religieux local et les sociabilités qui en découlent. Selon ses propres termes, «une des fonctions de la confraternité est sans doute aussi à Venise de faire participer ses membres à l'universalité de la ville ${ }^{57}$, même si parallèlement, l'appartenance à la communauté paroissiale demeure importante à travers l'élection de sépulture et surtout à travers les dons et les messes prescrites par les testateurs. L'équilibre entre la paroisse, le quartier et la cité dépend alors de chaque individu ${ }^{58}$. À cette triple échelle viennent se superposer ici le métier et l'appartenance nationale. Les confréries, et plus largement les «gestes dévotionnels » dont les testaments témoignent, sont donc des moyens d'exprimer des appartenances à plusieurs niveaux : appartenance à la cité dans son universalité, quitte à adhérer à des confréries lointaines parfois plus prestigieuses; appartenance à la paroisse et au quartier; enfin appartenance à une communauté nationale dans le cas d'étrangers immigrés à Venise. Nicolas de Francfort, à travers son adhésion à ces très nombreuses scuole, semble ici tirer parti de toutes les possibilités d'insertion qu'elles offrent, à différentes échelles géographiques et sociales.

\section{Un ancrage familial à Venise}

L'implantation de Nicolas de Francfort à Venise et dans la société vénitienne passe aussi par sa famille et ses choix d'alliances. De sa première femme, nous ne savons rien ou presque, si ce n'est sa pierre tombale à SS. Giovanni e Paolo ${ }^{59}$. En revanche, nous connaissons bien la seconde belle-famille de Nicolas de Francfort par les testaments qu'ont laissés sa femme, Andriana $^{60}$, et sa belle-mère, Gasparina ${ }^{61}$. Nous disposons également du testament du beaufils de Nicolas de Francfort, Jacomo ${ }^{62}$. À partir de ces documents, qui fournissent des informations jusqu'aux petits-enfants de Nicolas de Francfort, il est possible de comprendre en quoi ces liens familiaux ont pu contribuer à asseoir sa position et celle de sa famille dans l'élite artisanale vénitienne.

Nicolas de Francfort s'est donc remarié à une certaine Andriana ${ }^{63}$, fille de Gasparina ${ }^{64}$ et de Jacobo di Cha da Mestre de Murano, verrier. Celui-ci est inscrit plusieurs fois sur des listes de patrons verriers possédant un four et employant des ouvriers dans la ville ${ }^{65}$. Il est donc citoyen de Venise ou de Murano, puisque depuis 1482, les représentants de l'art demandent que les

\footnotetext{
57 Élisabeth Crouzet-Pavan, «Sopra le Acque salse » espaces, pouvoir et société à Venise à la fin du Moyen Âge, op. cit., p. 587.

$58 \quad$ Ibidem, pp. 589-590.

59 Henry Simonsfeld, Der Fondaco dei Tedeschi in Venedig und die Deutsch-Venetianischen Handelsbeziehungen, op. cit., vol. 2, $\mathrm{n}^{\circ} 63$ : «Pasche coniug. cariss. immatura morte extincte Nicolaus Fracforie maritus mestiss. suis et sibi V F an MCCCLXXXI».

60 Le 22 mai 1519, ASV, Notarile, Testamenti, b. 66, $\mathrm{n}^{\circ} 12$.

$61 \quad$ Le 21 septembre 1515, ASV, Notarile, Testamenti, b. 66, n 182.

62 Le 13 avril 1528, ASV, Cancelleria inferiore, Miscellanea, b. 29, n 2950.

63 ASV, Notarile, Testamenti, b. 66, $\mathrm{n}^{\circ} 12$.

64 ASV, Notarile, Testamenti, b. 66, $\mathrm{n}^{\circ} 182$

65 Luigi Zecchin, Vetro e vetrai di Murano. Studi sulla Storia del vetro, Venise, Arsenal Venise, 1987, vol. 3, p. 58 .
} 
patrons de four soient citoyens de Murano ou au moins de Venise ${ }^{66}$. Son aïeul Nicolo da Cha Mestre (v.1375-v.1450) est à l'origine de la fortune verrière familiale et la famille compte des gardiens de l'art ${ }^{67}$. Il s'agit donc d'une famille aisée qui occupe une place importante dans la communauté des verriers de Murano. Ils sont en particulier propriétaires immobiliers, puisque dans son testament, Gasparina lègue à sa fille et à son beau-fils une maison dans la paroisse de S. Stefano à Murano, ainsi que d'autres maisons à Marieta, sa deuxième fille ${ }^{68}$.

Son mariage avec Andriana da Cha da Mestre a permis à Nicolas de Francfort d'accéder au milieu vénitien et muranese des riches artisans; les verriers de Murano constituaient une catégorie aisée et d'un statut social enviable étant donné l'importance qu'ils revêtaient pour l'économie et le prestige de Venise. Cependant ce mariage n'est pas seulement une affaire d'argent. Nicolas de Francfort semble avoir eu une relation de confiance avec son épouse, comme en témoignent les legs et les responsabilités qu'il lui laisse après sa mort : elle obtient toute liberté pour mener à bien les affaires de son mari défunt. Enfin, des notes d'affection rares figurent dans le testament de l'imprimeur ; celles-ci nous apprennent en particulier qu'ils vivaient ensemble depuis trente-huit ans - donc depuis $1486^{69}$. Cette vie commune ne doit donc pas être réduite à une froide stratégie matrimoniale, économique et sociale, même si les effets ne doivent pas en être sous-estimés.

Le mariage de sa fille unique, Paula, à Jacomo dala Fontana va dans le même sens, même si on ne sait rien de l'origine du mari. Ce dernier est mentionné comme spicier dans la mariegola de S. Marco ${ }^{70}$. Ses biens semblent particulièrement étoffés : dans sa déclaration de patrimoine immobilier et foncier pour les Decime en 1514, il dit posséder des terres sur la Terre ferme, en particulier dans la région de Trévise, une maison dans la contrada de S. Bartolomeo à Venise, et une caxeta dans la contrada de S. Maria Nova ${ }^{71}$. Il est à noter que cet enrichissement a pu être récent, puisque Jacomo était inscrit sur les listes de la scuola de S. Marco parmi les confrères ala disciplina, c'est-à-dire la masse de confrères moins fortunés. Nicolas de Francfort, nouveau venu à Venise, marie sa fille à un homme dont la fortune marchande s'est apparemment construite au tournant du $\mathrm{XV}^{\mathrm{e}}$ siècle ; le soutien a dû être réciproque pour ces deux individus qui ne faisaient pas de droit partie de l'élite urbaine de Venise.

Si Paula semble malgré tout avoir fait un beau mariage, on en voit les effets sur ses enfants, les petits-enfants de Nicolas de Francfort, dont on sait pour l'un d'entre eux qu'il est doctor ; pour l'une des filles, Appolonia, il est certain qu'elle trouvera un mari convenable avec une dot de 800 ducats, qui représente une somme élevée pour un milieu d'artisans et de boutiquiers $^{72}$.

Les alliances matrimoniales de Nicolas de Francfort et de sa fille ont donc permis à leur famille de prospérer et de s'intégrer pleinement dans la société vénitienne, même si cette

\footnotetext{
66 Élisabeth Crouzet-Pavan, Les Villes vivantes : Italie XIII ${ }^{e}-X V^{e}$ siècles, Paris, Fayard, 2009, p. 257.

$67 \quad$ Luigi Zecchin, Vetro e vetrai di Murano. op. cit., p. 35.

68 ASV, Notarile, Testamenti, b. 66, $\mathrm{n}^{\circ} 182$.

69 ASV, Cancelleria inferiore, Miscellanea, b. 29, $\mathrm{n}^{\circ} 2936$ : «Quicquid dimitto dicte uxori mee sit pro satisfactione dotis sue et superabundans pro amore suo et caritate in me ac optima societate qua me semper est persecuta iam anno triginta octo ».

$70 \quad$ ASV, Scuola Grande di S. Marco, Atti, b. 4, Mariegola 1480-1549, f ${ }^{\circ}$ 68r : « Jachomo di bassani spicier ala fontana a san Bartolomeo. Mori marzo $1529 »$.

$71 \quad$ ASV, Savi Decime - 1514, b. $24, \mathrm{n}^{\circ} 4$.

72 ASV, Cancelleria inferiore, Miscellanea, b. $29, \mathrm{n}^{\circ} 2950$.
} 
intégration est notamment passée par un mariage avec une fille de verriers de Murano et un autre avec un - nouveau ? - riche marchand d'épices. Dans les deux cas, cela a permis une ouverture sur le monde artisanal et commerçant en dehors du monde du livre et des cercles allemands plus restreints. On peut expliquer ces alliances assez inhabituelles par le statut d'étranger de Nicolas de Francfort, qui n'a pas encore accumulé assez de capital social pour pouvoir se marier avec l'élite patricienne de Venise ${ }^{73}$. Elles témoignent également d'une volonté d'élargir son assise sociale au-delà du monde du livre. Il s'agit d'une stratégie différente de celle que l'on trouve à la même époque qui consiste à conclure des alliances matrimoniales au sein du milieu des typographes afin de consolider l'entreprise familiale et de permettre de conserver le capital en famille ${ }^{74}$. Alors même que Nicolas de Francfort a une production typographique épisodique, il ne cherche pas à s'ancrer davantage dans le monde du livre en y tissant des liens familiaux. Au contraire, il cherche des alliances qui lui permettent de s'insérer dans la société vénitienne et d'assurer par la même occasion son statut social et sa richesse ainsi que ceux de sa famille.

\section{Une diversification des intérêts économiques en direction du foncier et de la Terre ferme}

Son mariage avec Andriana da Cha da Mestre a également permis à Nicolas de Francfort d'accéder à la propriété foncière sur l'île de Murano. Dans sa déclaration de patrimoine foncier et immobilier pour les Decime, le 23 août 1514, on trouve par contre bien deux maisons dans la contrada de $\mathrm{S}$. Stefano à Murano ${ }^{75}$. La description de ces maisons révèle une aisance certaine ${ }^{76}$. Nicolas de Francfort, après son mariage avec Andriana et la mort de son beau-père, a sans doute pris en charge les propriétés de sa belle-mère, même si celle-ci en reste nominalement la propriétaire et peut en disposer dans son testament. Dès 1489, il est impliqué dans des conflits qui l'opposent à divers membres de la communauté de l'île ${ }^{77}$. Il cherche également à investir dans l'immobilier de l'île mais avec quelques difficultés puisque le 19 septembre 1493, le conseil des Quinze Sages du Rialto annule la vente de deux maisons à Nicolas de Francfort ${ }^{78}$. Il est possible que la communauté de Murano n'ait pas vu d'un bon oil cet imprimeur d'origine allemande chercher à s'y implanter.

Ses propriétés urbaines ne se limitent pas à l'apport de sa belle-famille : il possède également sa maison à S. Marziale, dans laquelle il vit en 1514. Son dernier testament parle d'une boutique qui pouvait être située à l'emplacement même de son logement. Cependant, avec son installation dans le quartier de S. Marziale, plus éloigné du centre libraire de Venise, Nicolas de Francfort semble avoir pris une certaine distance envers le monde du livre.

\footnotetext{
73 Ce genre de stratégie peut à terme aboutir à des alliances avec le patriciat, comme le montre le mariage de la petite-fille de Jean de Spire, premier imprimeur à Venise, avec Giovanni Barbarigo, membre d'une des plus importantes familles patriciennes de Venise. Ce cas a été notamment relevé par Philippe Braunstein, «Les Allemands et la naissance de l'imprimerie vénitienne », art. cit., p. 389.

$74 \quad$ Ainsi, Alde Manuce épouse la fille de son partenaire, l'imprimeur Andrea Torresanus ; Cristina, fille de Franz Renner, se marie à un autre imprimeur vénitien, Paganino de Paganini.

$75 \quad$ ASV, Savi Decime - 1514, b. 42, nº 63.

76 Élisabeth Crouzet-Pavan, «Murano à la fin du Moyen Âge : spécificité ou intégration dans l'espace vénitien ? », Revue historique, 1984, vol. CCLXVIII, n 1 , pp. 45-92.

77 Dans le cadre d'un conflit contre le prieur de S. Giovanni Battista de Murano, ASV, Podestà di Murano, b. 33, fasc. I, 31 août 1489 puis fasc. II, c 166, $1^{\text {er }}$ juillet 1490, où on voit que les deux parties sont parvenues à un compromis. Dans le cas d'un conflit contre Johannes Baroverio, ASV, Podestà di Murano, b. 35, 18 juillet 1493 ; le 29 juillet 1493, le podestat intime à Marco Dragano et Luigi Trevisan l'ordre de libérer la maison de Nicolas de Francfort. Ces trois derniers personnages appartiennent à des familles de patrons verriers.

$78 \quad$ ASV, Podestà di Murano, b. 35, 19 septembre 1493.
} 
Cette hypothèse est corroborée par le fait que Nicolas de Francfort s'est également lancé dans des investissements fonciers en terre ferme. Il possède des terres dans la villa de Pier Anzolo Lauro Vendramin ${ }^{79}$; il s'agit sans doute des terres qui sont mentionnées en 1495 à Preganziol, dans le Trévisan ${ }^{80}$. Suivant en cela le modèle des patriciens et de l'élite urbaine vénitienne, il en retire une rente régulière.

Ainsi, dès le début des années 1490, Nicolas de Francfort se lance dans une diversification de ses intérêts économiques en investissant dans le foncier et l'immobilier. Ces investissements correspondent au début de son mariage avec Andriana da Cha da Mestre et à un moment où sa production éditoriale ou typographique ralentit de façon significative : cette diversification signale donc certainement une volonté de s'assurer une rente par les loyers qu'il retire de ses possessions foncières et immobilières, à un moment où peut-être son âge ne lui permet plus de gérer une entreprise typographique, éditoriale et libraire comme auparavant.

Cette diversification n'est pas exceptionnelle dans le milieu de l'imprimerie vénitienne et les investissements fonciers en terre ferme se retrouvent fréquemment chez les plus importants imprimeurs du début du XVI ${ }^{\mathrm{e}}$ siècle $^{81}$; ils suivent en cela les mouvements d'investissements fonciers de l'élite patricienne et citoyenne vénitienne en terre ferme, commencée depuis le milieu du XV ${ }^{\mathrm{e}}$ siècle. Mais dans le cas de Nicolas de Francfort, cela s'accompagne d'un retrait relatif de l'activité typographique, le foncier et l'immobilier se substituant à terme en partie à l'imprimerie. Il conserve bien une boutique, sans doute de livres, mais il semble que ce ne soit plus son activité principale. Il s'agit d'une stratégie de diversification de ses revenus mais aussi de renforcement de son assise territoriale à Venise et dans les territoires sous sa domination. Le statut de Nicolas de Francfort en tant qu'Allemand à Venise tend donc à être moins net à la fin de sa vie : marié avec une fille de citoyen de Murano, propriétaire de terres et de maisons à Venise, à Murano et en terre ferme, il semble avoir dépassé sa condition d'étranger à sa mort en 1524.

\section{UN ALLEMAND A VENISE, OU VENITIEN ALLEMAND?}

\section{Des ponts coupés avec l'Allemagne?}

Le second testament de Nicolas de Francfort en 1524 est le dernier acte le mentionnant de son vivant ; très détaillé, il permet de faire un état des lieux de sa situation au seuil de sa mort, à la suite d'une longue vie - il est présent et actif à Venise depuis 52 ans. Son testament est intéressant à plusieurs titres. On en a évoqué certains aspects, mais il témoigne surtout d'une grande absence : des références à son origine allemande. Cette absence se retrouve d'abord dans le nom du testateur. Philippe Braunstein avait déjà remarqué pour le testament de 1519 que Nicolas de Francfort avait italianisé son nom en Nicolo Francoforte, dans un testament rédigé en langue vulgaire ${ }^{82}$; c'est en effet cette forme, en un seul ou en deux mots, que l'on retrouve dans de nombreux actes le concernant, notamment les actes du Podestat de Murano.

79 ASV, Savi Decime - 1514, b. 42, n 63 : «Campi 20 di terra vel circa in la villa di Pier Anzolo Lauro ser Vendramin ».

$80 \quad$ ASV, Giudici dell'esaminador, b. 45, fo 65v, 3 juillet 1495.

81 Par exemple, Paganino de Paganini possède des terres dans le Frioul, près de la rivière de Salo et une maison dans la paroisse de S. Salvador à Venise : Angela Nuovo, Alessandro Paganino : 1509-1538, Padoue, Antenore, 1990, pp. 242-246.

$82 \quad$ Philippe Braunstein, «Appunti per la storia di una minoranza : la popolazione tedesca di Venezia nel Medioevo », art. cit., p. 515. et ASV, Notarile, Testamenti, b. 66, $\mathrm{n}^{\circ} 320$. 
En 1524, dans un testament en latin cette fois, Nicolas de Francfort est nommé Nicolaus Franchfort $^{83}$. Si le nom de Francfort est moins italianisé que dans le précédent, il est cependant notable que dans l'un comme l'autre, Franchfort ou Francoforte est véritablement traité comme un nom de famille et non comme un surnom toponymique comme cela pouvait être le cas dans les colophons ${ }^{84}$. De plus, Nicolas de Francfort n'est plus qualifié d'alemanus, de germanus ou de teutonicus.

Son origine allemande n'est pas davantage visible dans le corps de l'acte. Tous les membres de sa famille qui y sont mentionnés ont des noms italiens et vivent très probablement à Venise. Contrairement à beaucoup d'imprimeurs étrangers installés à Venise dans les premiers temps de l'imprimerie, Nicolas de Francfort ne mentionne aucun parent resté en Allemagne, aucune propriété en dehors du territoire vénitien, aucun don à une église de sa ville d'origine. Un seul individu d'origine allemande figure parmi les exécuteurs testamentaires, les témoins et les légataires, et encore est-ce Peter Liechtenstein, imprimeur allemand mais installé à Venise depuis longtemps. Certes, les testaments ne représentent qu'une vue partielle de la vie d'un individu. Cependant, ce testament donne bien l'impression que la vie de Nicolas de Francfort s'est toujours déroulée à Venise et qu'il ne connaît que des Vénitiens. Il est possible qu'il soit arrivé à Venise très jeune, dans des circonstances que nous ignorons, qui ont sans doute fait qu'il n'avait plus que de faibles contacts avec sa famille ou son pays, si ce n'est par le biais de la colonie allemande de Venise.

Le testament qu'il laisse à sa mort donne véritablement le portrait d'un Vénitien. Il veut être enterré à SS. Giovanni e Paolo accompagné de la scuola de S. Marco dont il fait partie, il laisse quantité de legs à des scuole, à des institutions religieuses ou charitables de la ville et il lègue à sa femme l'argent placé dans les Monti de Venise. Cependant, des signes imperceptibles permettent de voir le maintien d'une «identité » allemande ${ }^{85}$, ou plutôt d'une appartenance à une communauté allemande à Venise. Il appartient tout d'abord à la confrérie nationale des Allemands, celle du Rosaire à $\mathrm{S}$. Bartolomeo, ainsi qu'à d'autres confréries où les Allemands sont très présents, notamment celles de S. Salvador. Ses relations avec Peter Liechtenstein, son compère et exécuteur testamentaire, le rattachent également à cette communauté. Plus qu'une identité allemande, qu'un sentiment d'appartenance à une patrie commune, il s'agit plutôt ici du maintien d'une sociabilité qui le rattache à la colonie allemande vénitienne, malgré le fait que ses liens sociaux plus récents, à travers sa famille notamment, se soient développés en dehors de ce cadre. Ici, les relations avec les pays germaniques ne sont plus qu'indirectes, à travers les Allemands résidant ou de passage à Venise. La question n'est finalement pas de savoir si Nicolas de Francfort se sentait Allemand ou Vénitien, mais la manière dont il pouvait investir cette double appartenance - si tant est que l'on puisse parler d'appartenance -, en pratique, dans ses actes de la vie quotidienne ou dans ses tentatives pour construire une entreprise et consolider sa position à Venise.

\footnotetext{
83 ASV, Cancelleria inferiore, Miscellanea, b. 29, n 2936.

$84 \quad$ Nicolas de Francfort y était alors appelé Nicolaus de Francfort ou le plus souvent Nicolaus de Franckfordia. On ne trouve qu'une seule occurrence d'un Nicholaus Franckforth en 1500, dans l'édition de Sermons qu'il effectue avec Bonetus Locatellus.

85 Sur les problèmes de l'emploi du terme «identité » en sciences humaines et sociales, voir Pierre Bourdieu, «L'Identité et la Représentation », Actes de la recherche en sciences sociales, vol. 35, 1980, pp. 6372 ; Rogers Brubacker et Frederick Cooper, «Beyond "Identity" », Theory and Society, n² 29, 2000, pp. 1-47 ; Martina Avanza et Gilles Laferté, «Dépasser la "Construction des identités" ? Identification image sociale, appartenance », Genèses, n 61, 2005, pp. 134-152.
} 


\section{Un Vénitien allemand entre singularité et exemplarité}

Nicolas de Francfort a su utiliser tant l'une que l'autre de ces communautés, en mettant à profit ses relations sociales. Sa volonté délibérée de sortir du strict cadre du monde du livre ou de la colonie allemande n'a pas pour autant rompu les liens qu'il pouvait entretenir au sein de ces deux milieux. La singularité de son parcours réside sans doute dans la multiplication de ses points d'ancrage dans la société vénitienne mais, pour autant, cela ne veut pas dire qu'il relève totalement de l'inhabituel. Pour les imprimeurs étrangers, les émigrés de première génération présents à Venise, il s'agit dans tous les cas d'utiliser tantôt les liens plus faciles à créer et parfois préexistants avec une communauté nationale et ceux, plus longs à instaurer, avec la société d'accueil.

Ce sont les structures sociales de Venise à la fin $d u X V^{e}$ siècle et au début du XVI siècle qui ont permis ce jeu de relations sociales que l'on voit illustrées ici par Nicolas de Francfort. À travers l'institution du Fondaco, qui est propre à la colonie allemande, mais également à travers l'autorisation de confréries nationales, les autorités vénitiennes ont permis la construction d'une forte cohésion de cette colonie étrangère dans la société urbaine. Cette communauté, dont les liens très étroits ont été renforcés par une culture et une langue proches et par ces occasions de sociabilité autorisées voire favorisées par le pouvoir vénitien, a donc pu être un terreau fertile pour l'implantation de la nouvelle industrie de l'imprimerie.

Mais dans le même temps, la ville a permis l'intégration de ces étrangers dans des institutions proprement vénitiennes. On a parlé des scuole, notamment des scuole grandi, où seules les fonctions de gouvernement de la confrérie sont réservées aux citoyens, mais où pour le reste, les étrangers peuvent être admis. Les possibilités d'alliances économiques, de mariages ou d'acquisition de propriétés foncières et immobilières sont également largement ouvertes aux étrangers, qui peuvent alors utiliser ces leviers pour contribuer à leur intégration dans la société vénitienne. L'implantation importante d'étrangers à Venise au cours du Moyen Âge et au tournant de l'époque moderne est largement favorisée par l'ouverture de la société, au-delà même de ses institutions.

En effet, les autorités peuvent selon les circonstances se montrer plus ou moins fermées en cas de surplus de main-d'œuvre et dans des sursauts récurrents mais rarement permanents de protectionnisme. Les étrangers restent pourtant là, même dans ces moments de fermeture institutionnelle, et ils évoluent tant dans leur communauté nationale que dans la société vénitienne dans son ensemble. Certains tendent alors vers une dissipation progressive de leur statut d'étranger, sinon juridiquement, du moins dans les esprits et dans les faits. L'acquisition de la citoyenneté vénitienne pouvait être recherchée pour des raisons économiques, car elle octroyait des privilèges commerciaux ${ }^{86}$. Mais souvent, en particulier chez les artisans, les étrangers devenaient Vénitiens sans en acquérir à proprement parler la citoyenneté, simplement parce que plus personne ne les considère ou ne les traite comme des étrangers. Ils deviennent Vénitiens au même titre que la foule du peuple vénitien exclue du statut de citoyen. Cette ouverture a favorisé l'implantation de l'imprimerie à Venise, permettant à des

86 On a pu voir ces cas de restriction pour les verriers de Murano, étudiés par Élisabeth Crouzet-Pavan. Sur le statut juridique des étrangers à Venise, voir en particulier Giorgio Fedalto, «Le minoranze straniere a Venezia tra politica e legislazione », dans Venezia, centro di mediazione tra Oriente e Occidente (secoli XV$X V I)$ : aspetti e problemi, op. cit., pp. 143-162, Reinhold C. Mueller, «Veneti facti privilegio : les étrangers naturalisés à Venise entre le XIV ${ }^{\mathrm{e}}$ et le XVI ${ }^{\mathrm{e}}$ siècle », in Jacques Bottin et Donatella Calabi (dir.), Les Étrangers dans la ville, Paris, Éditions MSH, 1999, pp. 171-181 et Giorgio Fedalto, « Stranieri a Venezia e a Padova », in Girolamo Arnaldi et Manlio Pastore Stocchi (dir.), Storia della cultura veneta, vol. 3, Vicence, Neri Pozza, 1980, pp. 499-535. 
étrangers de chercher des alliances dans la société vénitienne, sortant du cercle utile mais étroit des communautés nationales, plus spécifiquement de la colonie allemande.

Le cas de Nicolas de Francfort est emblématique d'une certaine catégorie d'artisans non vénitiens venus travailler à Venise, qui ont fini par faire oublier leur origine étrangère. Les choses étaient évidemment plus faciles avec les Italiens qui immigraient dans la Sérénissime ; la proximité culturelle et linguistique leur permettait de suivre plus aisément ces chemins de l'intégration. Cependant, le caractère d'extranéité est le même et certains entretiennent également une communauté nationale : ainsi les Milanais qui avaient également leur propre confrérie nationale.

En ce qui concerne l'imprimerie, la cohésion de la colonie allemande a permis un développement solide de certaines des plus grandes entreprises typographiques vénitiennes ; la pérennisation de cette industrie a nécessité un ancrage dans la ville par des alliances financières et par des liens matrimoniaux ou des sociabilités communes. Cependant, si ces mécanismes sont utiles pour comprendre l'implantation solide, rapide et exponentielle de l'imprimerie à Venise, ils sont également révélateurs de la manière dont la société vénitienne au tournant des $\mathrm{XV}^{\mathrm{e}}$ et $\mathrm{XVI}$ e siècles pouvait accueillir les nombreux étrangers qui s'y installaient. C'est un double mouvement de protection des nations étrangères en tant que communautés organisées et d'intégration dans la société vénitienne, qui dépasse donc largement les volontés épisodiques de protectionnisme, parce que les étrangers sont partout présents à Venise et parce que Venise a besoin des étrangers. 
ANNEXE

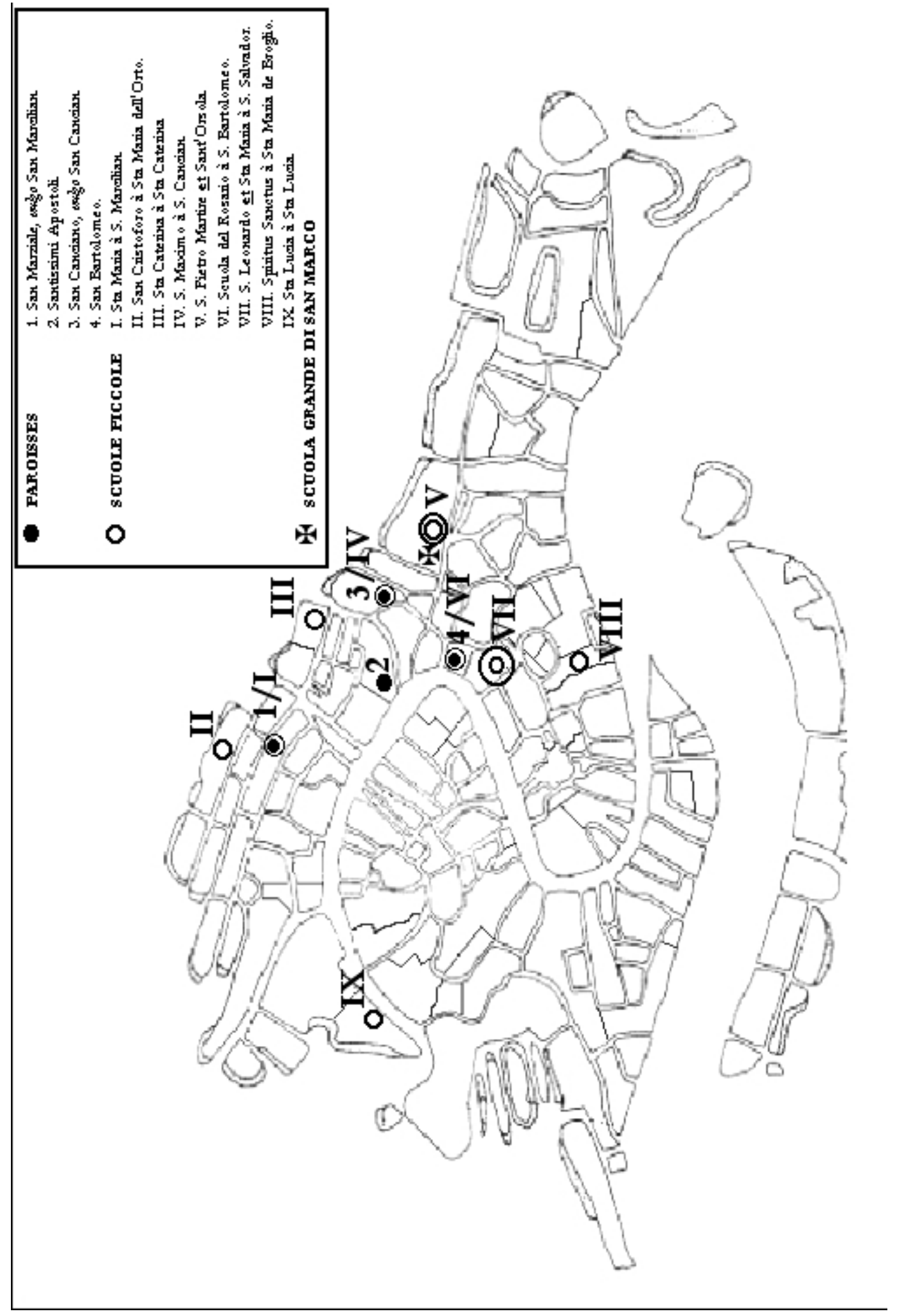


Catherine Kikuchi est doctorante allocataire monitrice à Paris IV-Sorbonne et prépare actuellement une thèse d'histoire médiévale sous la direction d'Élisabeth Crouzet-Pavan sur le thème des imprimeurs étrangers à Venise entre 1469 et le début du XVI siècle. Ses précédentes recherches avaient porté sur l'humanisme allemand et ses liens avec Venise, ce qui a donné lieu à la publication d'un article intitulé «La Bibliothèque de Hartmann Schedel à Nuremberg: les apports de Venise à l'humanisme allemand et leurs limites », Mélanges de l'École Française de Rome. Moyen Âge, 122/2, 2010, p. 379-391.

\section{RESUME EN FRANÇAIS}

Nicolas de Francfort (mort en 1524) est un imprimeur-éditeur d'origine allemande qui commence son activité à Venise en 1473. À travers le parcours de ce personnage mineur dans l'histoire de l'imprimerie, il est possible de comprendre le fonctionnement de ce milieu artisanal neuf, celui du livre imprimé à Venise entre la fin $d u X V^{\mathrm{e}}$ siècle et le début du $X V I^{\mathrm{e}}$ siècle, ainsi que la manière dont Venise intégrait les étrangers en son sein. La grande variété des sources documentaires conservées sur ce personnage nous permet une étude de cas précise. En tant qu'imprimeur-éditeur d'origine allemande, Nicolas de Francfort s'insère dans la colonie germanique bien présente et structurée à Venise; elle lui permet de nouer de fructueuses collaborations éditoriales. Mais les sources le concernant à Venise témoignent également d'une volonté de s'ancrer dans la ville par d'autres moyens, en particulier à travers ses nombreuses participations aux confréries ou scuole de la ville; ses alliances matrimoniales lui permettent de s'ouvrir aux autres milieux commerciaux et artisanaux de la ville; enfin il investit également dans le foncier à Venise et en terre ferme. Son parcours signale donc de la volonté de chercher une assise plus large au sein de la société vénitienne, au-delà du monde du livre. Il révèle également les mécanismes d'intégration des artisans étrangers à Venise qui, malgré une attitude parfois ambivalente des autorités, disposent d'un certain nombre de leviers pour construire une position économique et sociale stable et finissent par faire oublier leur statut d'étranger.

Mots clés

Moyen Âge, Venise, Imprimerie, Allemand, Étranger, Immigration, Artisanat.

ABSTRACT

Nicolas of Frankfurt (dead in 1524) is a German printer and publisher, who began his activity in Venice in 1473, shortly after the first book was printed in the city in 1469 by John of Spire. By analysing his life and career in Venice, we try to have a better understanding of the printing industry between the end of the $15^{\text {th }}$ century and the beginning of the $16^{\text {th }}$ century. Thanks to the great variety of sources - contracts, wills, judicial sources, epigraphy... -, we also aim to understand how foreign craftsmen were able to become integrate into the Venetian society. As a German printer and publisher, Nicolas of Frankfurt is not a major figure of history of printing. But he joined some lucrative and lasting associations within the German community in Venice and with other printers. Testimonies of his life in Venice also show that he tried to become integrated by other means and beyond the printing community. He participated to numerous confraternities or scuole, which is an important socialisation tool in the Venetian society. He also married the daughter of a glassblower from Murano and his 
daughter married a Venetian spice merchant. Finally, he made some investments in Venice real estate and in the Venetian terra ferma. His choices and alliances reveal some of the mechanisms of integration for a foreign craftsman in Venice, despite the sometimes ambiguous attitude of Venetian authorities. With this example, we try to show that foreign craftsmen, non-Italian as well as non-Venetian Italians, had tools to build themselves a stable socio-economical situation in the city, by using resources from their national community and by becoming an actor of the Venetian social life.

Keywords

Middle Ages, Venice, Printing, German, Foreigner, Immigration, Craft industry. 